\title{
Microbiological Analysis of Pharmaceutical Non Injectable Drugs Produced in Dhaka, Bangladesh
}

\author{
Moumita AB, Uddin MA and Ahmed T* \\ Department of Microbiology, Stamford University, Bangladesh
}

*Corresponding author: Tasnia Ahmed, Senior Lecturer, Department of Microbiology, Stamford University, Bangladesh, Tel: +8801784573970; Email: tasnia.ahmed@ stamforduniversity.edu.bd

\section{Research Article}

Volume 5 Issue 2

Received Date: March 16, 2020

Published Date: June 16, 2020

DOI: $10.23880 /$ oajmb-16000160

\section{Abstract}

Pharmaceutical drugs are applied and consumed by the patients with weak immune system and for this reason these products must be of good quality and within the required microbiological limit. Present study attempted to determine the microbiological quality of pharmaceutical non injectable oral and topical drugs as well as their antibacterial activity. A total of sixty samples were studied from different categories of medicine including syrup, tablet \& capsule and ointments. Microbiological analysis was done after serial dilution. Antibacterial activity of the samples was also determined by Kirby-Bauer method. The total viable bacterial count of 9 syrups, 7 tablet \& capsules and 13 ointments samples exceeded the microbial limit $<102 \mathrm{cfu} / \mathrm{ml}$ or cfu/gm recommended by USP (United States Pharmacopeia) and BP (British Pharmacopeia). Regarding to the presence of specific bacteria, about six, six and three samples from syrup, tablet \& capsule and ointment samples were of good quality respectively out of twenty samples each. Some drug prevailed good activity towards few bacteria and no activity at all to some others. As the drugs possess antibacterial activity, the contaminants might represent some other species of the same genera of bacteria having some mechanisms to prohibit such activities towards them. More than $50 \%$ of the drugs contain higher bacterial and fungal load rendering the quality at risk and not recommended to use by the patients to whom these products will impart most harm as these patients are already immune compromised.

Keywords: Pharmaceutical Drugs; Syrup; Ointments; Tablets; Contamination; Antibacterial Activity

Abbreviations: FDA: Food and Drug Administration; GMP: Good Manufacturing Practice; USP: United States Pharmacopeia; BP: British Pharmacopeia; NA: Nutrient Agar; TVC: Total Viable Count; USP: United States Pharmacopeia.

\section{Introduction}

To obtain the quality products in a pharmaceutical company, it is necessary to control all stages of drug production, which covers all matters influencing the quality of a product, including raw materials, the manufacturing process and the evaluation of finished product [1]. Primarily, the microbial quality of pharmaceutical products depends on the quality of raw materials, production process, production environment, hygiene of the personnel involved in manufacture and the storage conditions. Some other factors for contamination include faulty manufacturing and packaging process $[2,3]$. The presence of pathogenic microorganisms and the presence of relatively high number non-pathogenic microorganisms are objectionable in pharmaceutical products [4]. Among many types of pharmaceutical products, syrups are non- sterile liquid dosage which contain active medicaments and constitute the most convenient dosage for all ages but mostly prepared for oral administration in children since tablets and capsules cannot be easily or conveniently administered to them $[5,6]$. 


\section{Open Access Journal of Microbiology \& Biotechnology}

The United States Food and Drug Administration (FDA) require that drug product be tested for its purity, identity, strength, quality and stability before it can be released for use. Hence pharmaceutical validation and process control are important [7]. In non-sterile pharmaceutical preparations, the incidence of microbes is influenced by the nature of ingredients (raw materials), the quality of the vehicle, and the care and attitude of personnel involved in their handling including tablets oral dosage forms are not required to be sterile, but certain quality control measures are essential to keep the microbial content of these preparations safe and acceptable [8]. The extent of microbial contamination in tablets is usually influenced by the microbiological quality of the starting raw materials, packaging materials, personnel that come in contact with the product during the manufacturing process and the production environment as well as equipment. Failure to observe Good Manufacturing Practice (GMP) at any stage of production may consequently affect the microbiological quality of the product. Contaminants entrapped into the product via the above mentioned routes may not survive for a long period of time due to the lethal effect exerted by the various methods used in tablet production $[9,10]$. Basically, medicines get spoiled by initial or early pioneer invaders of biodegrading microorganisms, which prepare the way for later invaders, by degrading complex nutrients, altering the surrounding $\mathrm{pH}$ and making more moisture available [11,12]. Though pharmaceutical industries are growing fast in Bangladesh, the quality of drugs is being compromised due to unlicensed industries and competitive marketing [13]. So it is necessary to check microbiological quality of pharmaceutical products to assure consumer safety.

Therefore, microbiological assessment of the product and the knowledge of pathogen-specific antibiotic resistance are important. Similar studies were done for different pharmaceutical products in Bangladesh where on an average $50 \%$ products exceeded the microbial limit by USP (United States Pharmacopeia) and BP (British Pharmacopeia) with complete absence of Salmonella spp. [14,15]. But in current context we studied with a large sample volume to check if the condition has improved or not. Current study was attempted to understand the current scenario of non-injectable pharmaceutical products like syrup, tablet \& capsule and ointments along with antibacterial activity against some common microorganisms.

\section{Materials and Methods}

\section{Sample Collection and Preparation}

Seventy samples (Syrup-20, Tablet \& Capsule-20, Ointment-20) were collected from different types of medicinal stores. All samples were tested to assess the bacterial and fungal load as well as to detect the presence of specific pathogenic bacteria and actinomycetes, using the standard microbiological and biochemical methods [16-20].

\section{Dilution of Sample}

For homogenized sample, serial dilution of all the pharmaceutical samples was done individually. 10 fold serial dilutions were then prepared by transferring one $\mathrm{ml}$ of the original well homogenized sample to a tube containing $9 \mathrm{ml}$ of sterile normal saline and so forth until $10^{-5}$ dilution was obtained. Each test tube was labeled with the type of sample and number of the dilution.

\section{Enumeration of Total Viable Bacterial and Fungal Count}

An aliquot of $0.1 \mathrm{ml}$ of each suspension from the dilution $10^{-2}$ was spread onto nutrient agar (NA) plate and Sabouraud dextrose agar (SDA) plate for enumerating total viable count (TVC) and total fungal load respectively [21-26]. The plates were incubated at $37^{\circ} \mathrm{C}$ for 24 hours (NA plates) and at $25^{\circ} \mathrm{C}$ for 48 to 72 hours (SDA plates).

\section{Enumeration of Specific Pathogens}

From the dilution of $10^{-2}$ of each sample, $0.1 \mathrm{ml}$ of suspension was spread onto MacConkey agar, mannitol salt agar (MSA), cetrimide agar, and SS agar media for the enumeration of Escherichia coli, Klebsiella spp., Staphylococcus spp., Pseudomonas spp., Salmonella spp. and Shigella spp. consecutively. All the plates were incubated at $37 \mathrm{oC}$ for 24 hours. Presence of E. coli was further confirmed by the appearance of bluish-black colonies with green metallic sheen on the eosine-methylene blue (EMB) agar [24]. Confirmative biochemical tests were carried out for the final identification of the isolates $[16,20,24]$.

\section{Determination of Antimicrobial Activity of the Samples}

The antibacterial activity of the samples was performed following the agar well diffusion method $[27,28]$. The suspension of the samples was used directly on the MuellerHinton agar media. At first, the pathogenic bacterial suspensions with 0.5 McFarland turbidity (Pseudomonas spp, Vibrio spp, Salmonella spp, Klebsiella spp, Staphylococcus aureus, Proteus spp., Enterobacter spp., Acinetobacter spp.) were inoculated over the Mueller-Hinton agar media separately using cotton swab and $100 \mu \mathrm{l}$ of the suspension of drug samples were introduced into the wells made in the agar plates using sterile cork borer with a positive control 


\section{Open Access Journal of Microbiology \& Biotechnology}

(antibiotic disc) and a negative control (normal saline). Presence of clear zone around the wells filled with the sample solution indicated the antibacterial activity of tested samples.

\section{Results}

Much heterotrophic bacterial growth was observed within the pharmaceutical products in the current study. For syrup samples as well as tablet and capsule samples, the total bacterial count ranged from $1.0 \times 10^{2} \mathrm{cfu} /$ unit to $9.2 \times 10^{4} \mathrm{cfu} /$ unit (Tables $1 \& 2$ ). Ointment samples showed growth of total aerobic bacteria with lowest count in sample 18 of about $1.8 \times 10^{2} \mathrm{cfu} / \mathrm{gm}$ to $8.9 \times 10^{4} \mathrm{cfu} / \mathrm{gm}$ (Table 3) in sample 6 and 14 respectively. Raw materials also harbored aerobic bacteria ranging from $2.4 \times 10^{2} \mathrm{cfu} / \mathrm{gm}$ in milk of magnesia to $2.7 \times 10^{4} \mathrm{cfu} / \mathrm{gm}$ in magnesium chloride (Table
4). Escherichia coli was absent in all of the 70 samples. Most prevalent contaminants found in all of the products include Staphylococcus aureus (highest count in tablet \& capsules), Pseudomonas spp. (highest count in syrup samples as well as tablet \& capsule samples) and Klebsiella spp. (highest in sample 4 of tablet and capsule). Raw materials tested in this study were free from Salmonella spp. but some of the finished products like syrup, tablet \& capsule, ointments were found to be contaminated with Salmonella spp. Syrup samples 12 and 20 were free from specific organisms even though they contain higher total bacterial count. Total 6 samples out of $20(1,5,9,14,15,18)$ were found to be in approvable range for consumer safety compared to other samples. Sample no 6, 7, 8, 9, 10 and 18 of tablet \& capsule were under the recommended microbial limit $[16,29]$. Only 3 ointment samples were of good quality here (Tables $5 \& 6$ ).

\begin{tabular}{|c|c|c|c|c|c|c|c|}
\hline Sample & $\begin{array}{c}\text { TVB } \\
\text { (cfu/g) }\end{array}$ & $\begin{array}{l}\text { Total fun- } \\
\text { gal count }\end{array}$ & $\begin{array}{c}\text { E. coli } \\
\text { (cfu/ml) }\end{array}$ & $\begin{array}{c}\text { Klebsiella } \\
\text { spp. (cfu/ml) }\end{array}$ & $\begin{array}{c}\text { Salmonella } \\
\text { spp. (cfu/ ml) }\end{array}$ & $\begin{array}{c}\text { Pseudomonasspp. } \\
\text { (cfu/ml) }\end{array}$ & $\begin{array}{c}\text { Staphyloco- } \\
\text { cusspp. }(c f u / m l)\end{array}$ \\
\hline 1. Magmil & $3.5 \times 10^{2}$ & $2.5 \times 10^{1}$ & - & - & - & $2.0 \times 10^{2}$ & $5.3 \times 10^{2}$ \\
\hline 2. Benmet & $5.5 \times 10^{2}$ & $4.6 \times 10^{1}$ & - & $2.0 \times 10^{3}$ & - & $2.0 \times 10^{2}$ & $5.5 \times 10^{2}$ \\
\hline 3. Suzel & $2.0 \times 10^{2}$ & - & - & $3.3 \times 10^{3}$ & - & - & $3.0 \times 10^{2}$ \\
\hline 4. Megalax & $1.0 \times 10^{3}$ & - & - & - & - & - & $2.0 \times 10^{2}$ \\
\hline 5. Gavisol & $1.8 \times 10^{2}$ & - & - & $8.0 \times 10^{2}$ & - & - & $1.8 \times 10^{2}$ \\
\hline 6. Nactar & $1.6 \times 10^{2}$ & $3.3 \times 10^{1}$ & - & $1.0 \times 10^{2}$ & - & $1.1 \times 10^{3}$ & $3.0 \times 10^{2}$ \\
\hline 7. Cremag & $2.7 \times 10^{3}$ & - & - & $1.1 \times 10^{2}$ & - & $1.8 \times 10^{2}$ & $3.6 \times 10^{2}$ \\
\hline 8. Laxefin & $1.3 \times 10^{3}$ & $2.1 \times 10^{1}$ & - & $3.0 \times 10^{2}$ & $1.3 \times 10^{3}$ & $1.2 \times 10^{4}$ & - \\
\hline 9. Flustar & $1.8 \times 10^{2}$ & - & - & $6.6 \times 10^{2}$ & - & $1.0 \times 10^{2}$ & - \\
\hline 10. Cosy & $2.2 \times 10^{4}$ & - & - & $2.0 \times 10^{2}$ & - & $3.2 \times 10^{4}$ & - \\
\hline 11. Nitaxide & $1.0 \times 10^{2}$ & - & - & $2.7 \times 10^{2}$ & - & $1.3 \times 10^{3}$ & - \\
\hline 12. Anthel & $9.2 \times 10^{4}$ & - & - & - & - & - & - \\
\hline 13. Zesup & $2.5 \times 10^{2}$ & $1.3 \times 10^{1}$ & - & - & - & - & $1.0 \times 10^{3}$ \\
\hline 14. Visocid & $1.2 \times 10^{2}$ & $6.0 \times 10^{1}$ & - & - & $1.3 \times 10^{3}$ & - & $1.8 \times 10^{2}$ \\
\hline 15. Orsal & $6.8 \times 10^{2}$ & $5.2 \times 10^{1}$ & - & - & - & - & $1.6 \times 10^{2}$ \\
\hline 16. Bercef & $2.7 \times 10^{3}$ & $4.2 \times 10^{1}$ & - & $1.7 \times 10^{2}$ & - & $2.7 \times 10^{3}$ & $2.7 \times 10^{3}$ \\
\hline 17. Macorax & $1.3 \times 10^{3}$ & - & - & $7.0 \times 10^{3}$ & - & $1.3 \times 10^{3}$ & $1.3 \times 10^{3}$ \\
\hline 18. Magfin & $1.8 \times 10^{2}$ & - & - & $1.8 \times 10^{2}$ & - & - & $1.8 \times 10^{2}$ \\
\hline 19. Ceflon & $2.2 \times 10^{4}$ & $1.1 \times 10^{1}$ & - & $1.6 \times 10^{2}$ & - & - & $2.2 \times 10^{4}$ \\
\hline 20. Tuspel & $2.7 \times 10^{3}$ & $1.1 \times 10^{1}$ & - & - & - & - & - \\
\hline
\end{tabular}

$*$ TVB= Total Viable Bacteria

Table1: Prevalence of Pathogenic Microorganisms in Syrup. 


\section{Open Access Journal of Microbiology \& Biotechnology}

\begin{tabular}{|c|c|c|c|c|c|c|}
\hline Sample & $\begin{array}{c}\text { TVB } \\
\text { (cfu/g) }\end{array}$ & $\begin{array}{c}\text { Total } \\
\text { fungal } \\
\text { count }\end{array}$ & $\begin{array}{l}\text { E. coli } \\
\text { (cfu/g) }\end{array}$ & $\begin{array}{l}\text { Klebsiella spp. } \\
\quad \text { (cfu/g) }\end{array}$ & $\begin{array}{l}\text { Pseudomonas spp. } \\
\text { (cfu/g) }\end{array}$ & $\begin{array}{c}\text { Staphylococcus spp. } \\
\text { (cfu/g) }\end{array}$ \\
\hline 1. Metfo & $2.2 \times 10^{4}$ & $2.1 \times 10^{1}$ & - & $3.3 \times 10^{3}$ & - & $4.1 \times 10^{3}$ \\
\hline 2. Tramic & $1.0 \times 10^{2}$ & - & - & $5.8 \times 10^{2}$ & $3.9 \times 10^{3}$ & $1.3 \times 10^{3}$ \\
\hline 3. Imet & $9.2 \times 10^{4}$ & - & - & $2.7 \times 10^{3}$ & $6.8 \times 10^{2}$ & $6.8 \times 10^{2}$ \\
\hline 4. Aduvit & $9.2 \times 10^{4}$ & - & - & $9.7 \times 10^{4}$ & - & $2.7 \times 10^{3}$ \\
\hline 5. Poidone & $6.1 \times 10^{2}$ & - & - & - & $2.7 \times 10^{4}$ & $6.7 \times 10^{4}$ \\
\hline 6. Gaston & $4.3 \times 10^{2}$ & $3.3 \times 10^{1}$ & - & - & $1.3 \times 10^{3}$ & - \\
\hline 7. Comet & - & $4.2 \times 10^{1}$ & - & - & - & - \\
\hline 8. Cal D & - & $2.1 \times 10^{1}$ & - & - & - & - \\
\hline 9. Rabprazo & - & $2.2 \times 10^{1}$ & - & - & - & - \\
\hline 10. 1- VITA & - & - & - & - & - & - \\
\hline 11. AC PR & $1.2 \times 10^{2}$ & - & - & - & - & $2.7 \times 10^{3}$ \\
\hline 12. Arilol & $6.8 \times 10^{2}$ & - & - & $3.3 \times 10^{3}$ & - & $6.7 \times 10^{4}$ \\
\hline 13. Baclof & $3.2 \times 10^{2}$ & $1.3 \times 10^{1}$ & - & - & $1.3 \times 10^{3}$ & $8.9 \times 10^{4}$ \\
\hline 14. Caltrol & $1.2 \times 10^{2}$ & $6.0 \times 10^{1}$ & - & $2.7 \times 10^{3}$ & $6.8 \times 10^{2}$ & $3.6 \times 10^{4}$ \\
\hline 15. Amdova & $6.8 \times 10^{2}$ & $5.2 \times 10^{1}$ & - & $6.7 \times 10^{4}$ & $2.7 \times 10^{3}$ & $2.1 \times 10^{2}$ \\
\hline 16. Diatrol & $2.7 \times 10^{3}$ & - & - & & $6.7 \times 10^{4}$ & $3.7 \times 10^{2}$ \\
\hline 17. Lipigent & $1.3 \times 10^{3}$ & - & - & & - & - \\
\hline 18. Olmesan & $1.8 \times 10^{2}$ & - & - & & - & - \\
\hline 19. Atova & $2.9 \times 10^{4}$ & $1.1 \times 10^{1}$ & - & - & - & - \\
\hline 20. Lisinopril & $3.1 \times 10^{3}$ & $1.1 \times 10^{1}$ & - & - & - & - \\
\hline
\end{tabular}

$*$ TVB $=$ Total Viable Bacteria

Table 2: Prevalence of Pathogenic Microorganisms in Tablet \& Capsule.

\begin{tabular}{|l|c|c|c|c|c|c|}
\hline \multicolumn{1}{|c|}{ Sample } & TVB cfu/g) & $\begin{array}{c}\text { Total fungal } \\
\text { count }\end{array}$ & E. coli (cfu/g) & $\begin{array}{c}\text { Klebsiella spp. } \\
\text { (cfu/g) }\end{array}$ & $\begin{array}{c}\text { Pseudomonas } \\
\text { spp. (cfu/g) }\end{array}$ & $\begin{array}{c}\text { Staphylococcus } \\
\text { spp.(cfu/g) }\end{array}$ \\
\hline 1. E-Burn & $8.7 \times 10^{3}$ & - & - & - & - & - \\
\hline 2. Xzema & $1.3 \times 10^{3}$ & - & - & - & - & - \\
\hline 3. Cosmotrin & $6.8 \times 10^{2}$ & - & - & - & - & $4.0 \times 10^{2}$ \\
\hline 4. Avison & $2.7 \times 10^{3}$ & - & - & - & $2.7 \times 10^{4}$ & $2.8 \times 10^{2}$ \\
\hline 5. Neostan & $6.7 \times 10^{4}$ & $1.6 \times 10^{1}$ & - & - & $1.3 \times 10^{3}$ & $3.0 \times 10^{2}$ \\
\hline 6. Xencort & $8.9 \times 10^{4}$ & - & - & $8.9 \times 10^{2}$ & $3.8 \times 10^{2}$ & - \\
\hline 7. Cetaphil & $3.6 \times 10^{4}$ & - & - & $5.5 \times 10^{2}$ & $5.7 \times 10^{4}$ & $4.7 \times 10^{2}$ \\
\hline 8. Exovate & $2.1 \times 10^{2}$ & - & - & $3.0 \times 10^{2}$ & $1.3 \times 10^{3}$ & - \\
\hline 9. Gentosep & $3.7 \times 10^{2}$ & $2.2 \times 10^{1}$ & - & $2.4 \times 10^{2}$ & - & $2.0 \times 10^{2}$ \\
\hline 10. Nebanol & - & $1.1 \times 10^{1}$ & - & $1.2 \times 10^{3}$ & - & $4.7 \times 10^{2}$ \\
\hline 11. Miki-H & - & - & - & $3.0 \times 10^{2}$ & - & $1.2 \times 10^{2}$ \\
\hline 12. Remus & - & - & - & - & - & 2 \\
\hline
\end{tabular}




\begin{tabular}{|l|c|c|c|c|c|c|}
\hline 13. Usidin & $2.5 \times 10^{4}$ & $1.3 \times 10^{1}$ & - & - & - & $5.3 \times 10^{2}$ \\
\hline 14. Lomexin & $8.9 \times 10^{4}$ & $6.0 \times 10^{1}$ & - & - & - & - \\
\hline 15. Amela & $3.6 \times 10^{4}$ & $5.2 \times 10^{1}$ & - & $5.6 \times 10^{2}$ & - & - \\
\hline $\begin{array}{l}\text { 16. Emulsifying } \\
\text { ointment }\end{array}$ & $6.7 \times 10^{4}$ & $4.2 \times 10^{1}$ & - & $4.7 \times 10^{2}$ & - & - \\
\hline 17. Pevison & $1.3 \times 10^{3}$ & $2.1 \times 10^{1}$ & - & - & - & - \\
\hline 18. Pevaryl & $1.8 \times 10^{2}$ & - & - & - & - & - \\
\hline 19. Clopirox & $3.1 \times 10^{4}$ & - & - & - & - & - \\
\hline 20. Turboclav & $7.2 \times 10^{3}$ & - & - & - & - & - \\
\hline
\end{tabular}

$*$ TVB $=$ Total Viable Bacteria.

Table 3: Prevalence of Pathogenic Microorganisms in Ointments.

\begin{tabular}{|c|c|c|c|c|c|c|c|c|}
\hline Sample & $\begin{array}{l}\text { Vibrio } \\
\text { spp }\end{array}$ & $\begin{array}{c}\text { Salmonella } \\
\text { spp }\end{array}$ & $\begin{array}{c}\text { Pseudomonas } \\
\text { spp }\end{array}$ & $\begin{array}{c}\text { S. } \\
\text { aureus }\end{array}$ & $\begin{array}{l}\text { Proteus } \\
\text { spp. }\end{array}$ & $\begin{array}{c}\text { Klebsiella } \\
\text { spp }\end{array}$ & $\begin{array}{c}\text { Enterobacter } \\
\text { spp }\end{array}$ & $\begin{array}{c}\text { Acinetobacter } \\
\text { spp }\end{array}$ \\
\hline 1. Magmil & $13 \mathrm{~mm}$ & $0 \mathrm{~mm}$ & $13 \mathrm{~mm}$ & $0 \mathrm{~mm}$ & $0 \mathrm{~mm}$ & $0 \mathrm{~mm}$ & $11 \mathrm{~mm}$ & $11 \mathrm{~mm}$ \\
\hline 2. Benmet & $12 \mathrm{~mm}$ & $16 \mathrm{~mm}$ & $12 \mathrm{~mm}$ & $0 \mathrm{~mm}$ & $19 \mathrm{~mm}$ & $0 \mathrm{~mm}$ & $12 \mathrm{~mm}$ & $13 \mathrm{~mm}$ \\
\hline 3. Suzel & $0 \mathrm{~mm}$ & $0 \mathrm{~mm}$ & $0 \mathrm{~mm}$ & $23 \mathrm{~mm}$ & $13 \mathrm{~mm}$ & $0 \mathrm{~mm}$ & $0 \mathrm{~mm}$ & $0 \mathrm{~mm}$ \\
\hline 4. Megalax & $10 \mathrm{~mm}$ & $10 \mathrm{~mm}$ & $10 \mathrm{~mm}$ & $17 \mathrm{~mm}$ & $0 \mathrm{~mm}$ & $12 \mathrm{~mm}$ & $0 \mathrm{~mm}$ & $13 \mathrm{~mm}$ \\
\hline 5. Gavisol & $12 \mathrm{~mm}$ & $0 \mathrm{~mm}$ & $12 \mathrm{~mm}$ & $0 \mathrm{~mm}$ & $0 \mathrm{~mm}$ & $14 \mathrm{~mm}$ & $12 \mathrm{~mm}$ & $15 \mathrm{~mm}$ \\
\hline 6. Nactar & $15 \mathrm{~mm}$ & $0 \mathrm{~mm}$ & $15 \mathrm{~mm}$ & $0 \mathrm{~mm}$ & $15 \mathrm{~mm}$ & $15 \mathrm{~mm}$ & $0 \mathrm{~mm}$ & $0 \mathrm{~mm}$ \\
\hline 7. Cremag & $12 \mathrm{~mm}$ & $23 \mathrm{~mm}$ & $12 \mathrm{~mm}$ & $0 \mathrm{~mm}$ & $16 \mathrm{~mm}$ & $0 \mathrm{~mm}$ & $0 \mathrm{~mm}$ & $9 \mathrm{~mm}$ \\
\hline 8. Laxefin & $21 \mathrm{~mm}$ & $17 \mathrm{~mm}$ & $8 \mathrm{~mm}$ & $0 \mathrm{~mm}$ & $12 \mathrm{~mm}$ & $0 \mathrm{~mm}$ & $12 \mathrm{~mm}$ & $7 \mathrm{~mm}$ \\
\hline 9. Flustar & $20 \mathrm{~mm}$ & $0 \mathrm{~mm}$ & $0 \mathrm{~mm}$ & $14 \mathrm{~mm}$ & $0 \mathrm{~mm}$ & $12 \mathrm{~mm}$ & $14 \mathrm{~mm}$ & $9 \mathrm{~mm}$ \\
\hline 10. Cosy & $10 \mathrm{~mm}$ & $0 \mathrm{~mm}$ & $15 \mathrm{~mm}$ & 13 mm & $0 \mathrm{~mm}$ & $15 \mathrm{~mm}$ & $14 \mathrm{~mm}$ & $12 \mathrm{~mm}$ \\
\hline 11. Nitaxide & $25 \mathrm{~mm}$ & $0 \mathrm{~mm}$ & $0 \mathrm{~mm}$ & 18 mm & $0 \mathrm{~mm}$ & $16 \mathrm{~mm}$ & $12 \mathrm{~mm}$ & $8 \mathrm{~mm}$ \\
\hline 12. Anthel & $9 \mathrm{~mm}$ & $0 \mathrm{~mm}$ & $0 \mathrm{~mm}$ & $9 \mathrm{~mm}$ & $13 \mathrm{~mm}$ & $0 \mathrm{~mm}$ & $13 \mathrm{~mm}$ & $12 \mathrm{~mm}$ \\
\hline 13. Zesup & $17 \mathrm{~mm}$ & $14 \mathrm{~mm}$ & $15 \mathrm{~mm}$ & $0 \mathrm{~mm}$ & $17 \mathrm{~mm}$ & $12 \mathrm{~mm}$ & $15 \mathrm{~mm}$ & $14 \mathrm{~mm}$ \\
\hline 14. Visocid & $16 \mathrm{~mm}$ & $13 \mathrm{~mm}$ & $16 \mathrm{~mm}$ & $12 \mathrm{~mm}$ & $0 \mathrm{~mm}$ & $17 \mathrm{~mm}$ & $16 \mathrm{~mm}$ & $0 \mathrm{~mm}$ \\
\hline 15. Orsal & $19 \mathrm{~mm}$ & $18 \mathrm{~mm}$ & $12 \mathrm{~mm}$ & $11 \mathrm{~mm}$ & $0 \mathrm{~mm}$ & $0 \mathrm{~mm}$ & $0 \mathrm{~mm}$ & $8 \mathrm{~mm}$ \\
\hline 16. Bercef & $17 \mathrm{~mm}$ & $20 \mathrm{~mm}$ & $13 \mathrm{~mm}$ & 13 mm & $12 \mathrm{~mm}$ & $0 \mathrm{~mm}$ & $0 \mathrm{~mm}$ & $14 \mathrm{~mm}$ \\
\hline 17. Macorax & $0 \mathrm{~mm}$ & $17 \mathrm{~mm}$ & $16 \mathrm{~mm}$ & $0 \mathrm{~mm}$ & $14 \mathrm{~mm}$ & $0 \mathrm{~mm}$ & $0 \mathrm{~mm}$ & $0 \mathrm{~mm}$ \\
\hline 18. Magfin & $16 \mathrm{~mm}$ & $0 \mathrm{~mm}$ & $17 \mathrm{~mm}$ & $10 \mathrm{~mm}$ & $0 \mathrm{~mm}$ & $0 \mathrm{~mm}$ & $0 \mathrm{~mm}$ & $0 \mathrm{~mm}$ \\
\hline 19. Ceflon & $14 \mathrm{~mm}$ & $16 \mathrm{~mm}$ & $0 \mathrm{~mm}$ & $18 \mathrm{~mm}$ & $17 \mathrm{~mm}$ & $0 \mathrm{~mm}$ & $0 \mathrm{~mm}$ & $12 \mathrm{~mm}$ \\
\hline 20. Tuspel & $19 \mathrm{~mm}$ & $12 \mathrm{~mm}$ & $0 \mathrm{~mm}$ & $13 \mathrm{~mm}$ & $13 \mathrm{~mm}$ & $0 \mathrm{~mm}$ & $0 \mathrm{~mm}$ & $0 \mathrm{~mm}$ \\
\hline
\end{tabular}

Table 4: Antimicrobial Activity of Syrup Sample.

All of the twenty syrup samples showed some degree of antimicrobial properties against several bacterial isolates. The average zone of inhibition was $15 \mathrm{~mm}$. highest activity was showed against Vibrio spp. (Sample 8-21 mm, sample
9-20 mm, sample 11-25 mm) and Salmonella spp. (sample 7-23 $\mathrm{mm}$, sample 16-20 $\mathrm{mm}$ ). Antibacterial activity against other isolates showed similar results with syrup samples. 
Open Access Journal of Microbiology \& Biotechnology

\begin{tabular}{|l|c|c|c|c|c|c|c|c|}
\hline \multicolumn{1}{|c|}{ Sample } & $\begin{array}{c}\text { Vibrio } \\
\text { spp }\end{array}$ & $\begin{array}{c}\text { Salmonella } \\
\text { spp }\end{array}$ & $\begin{array}{c}\text { Pseudomonas } \\
\text { spp }\end{array}$ & $\begin{array}{c}\text { S. } \\
\text { aureus }\end{array}$ & $\begin{array}{c}\text { Proteus } \\
\text { Spp. }\end{array}$ & $\begin{array}{c}\text { Klebsiella } \\
\text { spp }\end{array}$ & $\begin{array}{c}\text { Enterobacter } \\
\text { spp }\end{array}$ & $\begin{array}{c}\text { Acinetobacter } \\
\text { spp }\end{array}$ \\
\hline 1. Metfo & $0 \mathrm{~mm}$ & $0 \mathrm{~mm}$ & $0 \mathrm{~mm}$ & $0 \mathrm{~mm}$ & $9 \mathrm{~mm}$ & $13 \mathrm{~mm}$ & $0 \mathrm{~mm}$ & $13 \mathrm{~mm}$ \\
\hline 2. Tramic & $15 \mathrm{~mm}$ & $0 \mathrm{~mm}$ & $0 \mathrm{~mm}$ & $0 \mathrm{~mm}$ & $23 \mathrm{~mm}$ & $13 \mathrm{~mm}$ & $0 \mathrm{~mm}$ & $0 \mathrm{~mm}$ \\
\hline 3. Imet & $0 \mathrm{~mm}$ & $0 \mathrm{~mm}$ & $10 \mathrm{~mm}$ & $10 \mathrm{~mm}$ & $17 \mathrm{~mm}$ & $0 \mathrm{~mm}$ & $12 \mathrm{~mm}$ & $0 \mathrm{~mm}$ \\
\hline 4. Aduvit & $10 \mathrm{~mm}$ & $15 \mathrm{~mm}$ & $0 \mathrm{~mm}$ & $12 \mathrm{~mm}$ & $0 \mathrm{~mm}$ & $0 \mathrm{~mm}$ & $14 \mathrm{~mm}$ & $12 \mathrm{~mm}$ \\
\hline 5. Poidone & $0 \mathrm{~mm}$ & $16 \mathrm{~mm}$ & $0 \mathrm{~mm}$ & $15 \mathrm{~mm}$ & $0 \mathrm{~mm}$ & $15 \mathrm{~mm}$ & $15 \mathrm{~mm}$ & $0 \mathrm{~mm}$ \\
\hline 6. Gaston & $15 \mathrm{~mm}$ & $12 \mathrm{~mm}$ & $23 \mathrm{~mm}$ & $12 \mathrm{~mm}$ & $0 \mathrm{~mm}$ & $16 \mathrm{~mm}$ & $0 \mathrm{~mm}$ & $0 \mathrm{~mm}$ \\
\hline 7. Comet & $11 \mathrm{~mm}$ & $0 \mathrm{~mm}$ & $17 \mathrm{~mm}$ & $8 \mathrm{~mm}$ & $0 \mathrm{~mm}$ & $12 \mathrm{~mm}$ & $0 \mathrm{~mm}$ & $12 \mathrm{~mm}$ \\
\hline 8. Cal D & $13 \mathrm{~mm}$ & $0 \mathrm{~mm}$ & $0 \mathrm{~mm}$ & $0 \mathrm{~mm}$ & $14 \mathrm{~mm}$ & $0 \mathrm{~mm}$ & $12 \mathrm{~mm}$ & $14 \mathrm{~mm}$ \\
\hline 9. Rabprazo & $14 \mathrm{~mm}$ & $0 \mathrm{~mm}$ & $0 \mathrm{~mm}$ & $15 \mathrm{~mm}$ & $13 \mathrm{~mm}$ & $0 \mathrm{~mm}$ & $15 \mathrm{~mm}$ & $14 \mathrm{~mm}$ \\
\hline 10. 1- VITA & $14 \mathrm{~mm}$ & $13 \mathrm{~mm}$ & $0 \mathrm{~mm}$ & $0 \mathrm{~mm}$ & $18 \mathrm{~mm}$ & $0 \mathrm{~mm}$ & $16 \mathrm{~mm}$ & $12 \mathrm{~mm}$ \\
\hline 11. AC PR & $15 \mathrm{~mm}$ & $0 \mathrm{~mm}$ & $0 \mathrm{~mm}$ & $0 \mathrm{~mm}$ & $9 \mathrm{~mm}$ & $13 \mathrm{~mm}$ & $0 \mathrm{~mm}$ & $13 \mathrm{~mm}$ \\
\hline 12. Arilol & $9 \mathrm{~mm}$ & $12 \mathrm{~mm}$ & $14 \mathrm{~mm}$ & $15 \mathrm{~mm}$ & $0 \mathrm{~mm}$ & $17 \mathrm{~mm}$ & $12 \mathrm{~mm}$ & $15 \mathrm{~mm}$ \\
\hline 13. Baclof & $17 \mathrm{~mm}$ & $0 \mathrm{~mm}$ & $13 \mathrm{~mm}$ & $16 \mathrm{~mm}$ & $12 \mathrm{~mm}$ & $0 \mathrm{~mm}$ & $17 \mathrm{~mm}$ & $16 \mathrm{~mm}$ \\
\hline 14. Caltrol & $0 \mathrm{~mm}$ & $12 \mathrm{~mm}$ & $18 \mathrm{~mm}$ & $12 \mathrm{~mm}$ & $11 \mathrm{~mm}$ & $0 \mathrm{~mm}$ & $0 \mathrm{~mm}$ & $0 \mathrm{~mm}$ \\
\hline 15. Amdova & $0 \mathrm{~mm}$ & $10 \mathrm{~mm}$ & $20 \mathrm{~mm}$ & $13 \mathrm{~mm}$ & $13 \mathrm{~mm}$ & $12 \mathrm{~mm}$ & $0 \mathrm{~mm}$ & $0 \mathrm{~mm}$ \\
\hline 16. Diatrol & $0 \mathrm{~mm}$ & $24 \mathrm{~mm}$ & $0 \mathrm{~mm}$ & $0 \mathrm{~mm}$ & $0 \mathrm{~mm}$ & $0 \mathrm{~mm}$ & $15 \mathrm{~mm}$ & $11 \mathrm{~mm}$ \\
\hline 17. Lipigent & $0 \mathrm{~mm}$ & $0 \mathrm{~mm}$ & $0 \mathrm{~mm}$ & $15 \mathrm{~mm}$ & $12 \mathrm{~mm}$ & $0 \mathrm{~mm}$ & $14 \mathrm{~mm}$ & $0 \mathrm{~mm}$ \\
\hline 18. Olmesan & $16 \mathrm{~mm}$ & $9 \mathrm{~mm}$ & $0 \mathrm{~mm}$ & $16 \mathrm{~mm}$ & $12 \mathrm{~mm}$ & $0 \mathrm{~mm}$ & $0 \mathrm{~mm}$ & $12 \mathrm{~mm}$ \\
\hline 19. Atova & $14 \mathrm{~mm}$ & $12 \mathrm{~mm}$ & $12 \mathrm{~mm}$ & $12 \mathrm{~mm}$ & $0 \mathrm{~mm}$ & $0 \mathrm{~mm}$ & $0 \mathrm{~mm}$ & $11 \mathrm{~mm}$ \\
\hline 20. Lisinopril & $19 \mathrm{~mm}$ & $11 \mathrm{~mm}$ & $12 \mathrm{~mm}$ & $0 \mathrm{~mm}$ & $0 \mathrm{~mm}$ & $0 \mathrm{~mm}$ & $0 \mathrm{~mm}$ & $0 \mathrm{~mm}$ \\
\hline
\end{tabular}

Table 5: Antimicrobial Activity of Tablet \& Capsule Sample.

With tablet and capsule sample, lowest number of samples had antimicrobial activities against Klebisella spp. just as like the syrup samples did. Highest effectivity was found with sample 2 for Proteus spp. (23 mm). Such high activity was also found for sample 6 and 15 against Pseudomonas spp. (23 $\mathrm{mm}$ and $20 \mathrm{~mm}$ respectively), sample 16 against Salmonella spp. (24 mm), and sample 20 against Vibrio spp. (19 mm).

\begin{tabular}{|l|c|c|c|c|c|c|c|c|}
\hline Sample & $\begin{array}{c}\text { Vibrio } \\
\text { spp }\end{array}$ & $\begin{array}{c}\text { Salmonella } \\
\text { spp }\end{array}$ & $\begin{array}{c}\text { Pseudomonas } \\
\text { spp }\end{array}$ & $\begin{array}{c}\text { S. } \\
\text { aureus }\end{array}$ & $\begin{array}{c}\text { Proteus } \\
\text { spp. }\end{array}$ & $\begin{array}{c}\text { Klebsiella } \\
\text { spp }\end{array}$ & $\begin{array}{c}\text { Enterobacter } \\
\text { spp }\end{array}$ & $\begin{array}{c}\text { Acinetobacter } \\
\text { spp }\end{array}$ \\
\hline 1. E-Burn & $0 \mathrm{~mm}$ & $0 \mathrm{~mm}$ & $0 \mathrm{~mm}$ & $13 \mathrm{~mm}$ & $16 \mathrm{~mm}$ & $0 \mathrm{~mm}$ & $12 \mathrm{~mm}$ & $0 \mathrm{~mm}$ \\
\hline 2. Xzema & $0 \mathrm{~mm}$ & $12 \mathrm{~mm}$ & $0 \mathrm{~mm}$ & $0 \mathrm{~mm}$ & $0 \mathrm{~mm}$ & $24 \mathrm{~mm}$ & $0 \mathrm{~mm}$ & $12 \mathrm{~mm}$ \\
\hline 3. Cosmotrin & $0 \mathrm{~mm}$ & $15 \mathrm{~mm}$ & $0 \mathrm{~mm}$ & $13 \mathrm{~mm}$ & $0 \mathrm{~mm}$ & $13 \mathrm{~mm}$ & $0 \mathrm{~mm}$ & $0 \mathrm{~mm}$ \\
\hline 4. Avison & $10 \mathrm{~mm}$ & $10 \mathrm{~mm}$ & $0 \mathrm{~mm}$ & $12 \mathrm{~mm}$ & $16 \mathrm{~mm}$ & $12 \mathrm{~mm}$ & $0 \mathrm{~mm}$ & $19 \mathrm{~mm}$ \\
\hline 5. Neostan & $12 \mathrm{~mm}$ & $12 \mathrm{~mm}$ & $16 \mathrm{~mm}$ & $0 \mathrm{~mm}$ & $0 \mathrm{~mm}$ & $0 \mathrm{~mm}$ & $23 \mathrm{~mm}$ & $13 \mathrm{~mm}$ \\
\hline 6. Xencort & $0 \mathrm{~mm}$ & $0 \mathrm{~mm}$ & $12 \mathrm{~mm}$ & $10 \mathrm{~mm}$ & $10 \mathrm{~mm}$ & $10 \mathrm{~mm}$ & $17 \mathrm{~mm}$ & $0 \mathrm{~mm}$ \\
\hline 7. Cetaphil & $0 \mathrm{~mm}$ & $12 \mathrm{~mm}$ & $0 \mathrm{~mm}$ & $12 \mathrm{~mm}$ & $0 \mathrm{~mm}$ & $0 \mathrm{~mm}$ & $0 \mathrm{~mm}$ & $0 \mathrm{~mm}$ \\
\hline 8. Exovate & $9 \mathrm{~mm}$ & $8 \mathrm{~mm}$ & $0 \mathrm{~mm}$ & $15 \mathrm{~mm}$ & $0 \mathrm{~mm}$ & $15 \mathrm{~mm}$ & $0 \mathrm{~mm}$ & $15 \mathrm{~mm}$ \\
\hline 9. Gentosep & $20 \mathrm{~mm}$ & $0 \mathrm{~mm}$ & $0 \mathrm{~mm}$ & $12 \mathrm{~mm}$ & $23 \mathrm{~mm}$ & $0 \mathrm{~mm}$ & $0 \mathrm{~mm}$ & $0 \mathrm{~mm}$ \\
\hline 10. Nebanol & $10 \mathrm{~mm}$ & $15 \mathrm{~mm}$ & $13 \mathrm{~mm}$ & $21 \mathrm{~mm}$ & $17 \mathrm{~mm}$ & $8 \mathrm{~mm}$ & $0 \mathrm{~mm}$ & $30 \mathrm{~mm}$ \\
\hline 11. Miki-H & $14 \mathrm{~mm}$ & $0 \mathrm{~mm}$ & $17 \mathrm{~mm}$ & $20 \mathrm{~mm}$ & $0 \mathrm{~mm}$ & $0 \mathrm{~mm}$ & $14 \mathrm{~mm}$ & $0 \mathrm{~mm}$ \\
\hline
\end{tabular}




\section{Open Access Journal of Microbiology \& Biotechnology}

\begin{tabular}{|l|c|c|c|c|c|c|c|c|}
\hline 12. Remus & $9 \mathrm{~mm}$ & $0 \mathrm{~mm}$ & $0 \mathrm{~mm}$ & $10 \mathrm{~mm}$ & $0 \mathrm{~mm}$ & $15 \mathrm{~mm}$ & $13 \mathrm{~mm}$ & $0 \mathrm{~mm}$ \\
\hline 13. Usidin & $15 \mathrm{~mm}$ & $15 \mathrm{~mm}$ & $0 \mathrm{~mm}$ & $25 \mathrm{~mm}$ & $0 \mathrm{~mm}$ & $0 \mathrm{~mm}$ & $18 \mathrm{~mm}$ & $0 \mathrm{~mm}$ \\
\hline 14. Lomexin & $0 \mathrm{~mm}$ & $16 \mathrm{~mm}$ & $12 \mathrm{~mm}$ & $9 \mathrm{~mm}$ & $0 \mathrm{~mm}$ & $0 \mathrm{~mm}$ & $9 \mathrm{~mm}$ & $13 \mathrm{~mm}$ \\
\hline 15. Amela & $0 \mathrm{~mm}$ & $12 \mathrm{~mm}$ & $0 \mathrm{~mm}$ & $17 \mathrm{~mm}$ & $14 \mathrm{~mm}$ & $15 \mathrm{~mm}$ & $0 \mathrm{~mm}$ & $17 \mathrm{~mm}$ \\
\hline $\begin{array}{l}\text { 16. Emulsifying } \\
\text { ointment }\end{array}$ & $17 \mathrm{~mm}$ & $13 \mathrm{~mm}$ & $0 \mathrm{~mm}$ & $16 \mathrm{~mm}$ & $13 \mathrm{~mm}$ & $16 \mathrm{~mm}$ & $12 \mathrm{~mm}$ & $0 \mathrm{~mm}$ \\
\hline 17. Pevison & $0 \mathrm{~mm}$ & $0 \mathrm{~mm}$ & $0 \mathrm{~mm}$ & $19 \mathrm{~mm}$ & $18 \mathrm{~mm}$ & $12 \mathrm{~mm}$ & $11 \mathrm{~mm}$ & $0 \mathrm{~mm}$ \\
\hline 18. Pevaryl & $16 \mathrm{~mm}$ & $0 \mathrm{~mm}$ & $0 \mathrm{~mm}$ & $17 \mathrm{~mm}$ & $20 \mathrm{~mm}$ & $13 \mathrm{~mm}$ & $13 \mathrm{~mm}$ & $12 \mathrm{~mm}$ \\
\hline 19. Clopirox & $14 \mathrm{~mm}$ & $15 \mathrm{~mm}$ & $0 \mathrm{~mm}$ & $0 \mathrm{~mm}$ & $17 \mathrm{~mm}$ & $16 \mathrm{~mm}$ & $0 \mathrm{~mm}$ & $14 \mathrm{~mm}$ \\
\hline 20. Turboclav & $19 \mathrm{~mm}$ & $0 \mathrm{~mm}$ & $12 \mathrm{~mm}$ & $16 \mathrm{~mm}$ & $0 \mathrm{~mm}$ & $17 \mathrm{~mm}$ & $10 \mathrm{~mm}$ & $0 \mathrm{~mm}$ \\
\hline
\end{tabular}

Table 6: Antimicrobial Activity of Ointment Sample.

Lowest antimicrobial activity was seen against Salmonella spp. and Pseudomonas spp. On an average better

\section{Discussion}

The purpose of this experiment was to determine the presence of microorganisms in finished pharmaceuticals products such as syrup, tablet \& capsule, ointment, raw materials etc. It is quite disappointing that all the products were contaminated with several kinds of bacteria and fungi as well. Total aerobic bacterial load was within $10^{4} \mathrm{cfu} / \mathrm{gm}$ or $\mathrm{cfu} / \mathrm{ml}$ and total fungal count did not exceed $10^{1} \mathrm{cfu} / \mathrm{gm}$ or $\mathrm{cfu} / \mathrm{ml}$. Other than these, Klebsiella spp., Pseudomonas spp., Staphylococcus aureus and even Salmonella spp. were present abundantly which were not supposed to be present to some samples like ointments. Nine syrup samples crossed the USP (United States Pharmacopeia) limit of total aerobic bacterial count of $<10^{2} \mathrm{cfu} / \mathrm{ml}$ making these products unsatisfactory to be used by patients. Topical ointments and aqueous preparations for oral use (syrups) must not contain Pseudomonas spp. and Staphylococcus aureus according to USP. The load of Klebsiella spp. must be not more than $10^{2}$ cfu/gm which already exceeded in several samples (3 syrup samples, 6 tablet $\&$ capsule samples). The non-aqueous oral drugs (tablet \& capsule) which were contaminated with Klebsiella spp., the load for all of these samples were more than the acceptable limit. The quality of the products is really at a stake to be used by the patients. As the pharmaceutical products under this study were not sterile products, they can have some bacteria but within a particular range of course. In addition to that there will be no pathogenic specific bacterial count which were already found to be present in the test samples $[16,29]$. As medicines are provided to the patients who are already diseased and in a immune suppressive condition due to the disease, administration of such contaminated drugs may cause a serious damage in them compared to healthy individuals. Samples can be contaminated in different ways in industrial sectors such as during handling of raw materials, water, processing of antibacterial activity was showed against Staphylococcus aureus, Proteus spp. and Klebsiella spp.

products, unclean machineries, contaminated packaging materials, storage condition of the packaging and raw materials etc. To ensure the safety of people it is necessary to identify the source of contamination related to such drugs. In industrial sectors, workers hygiene who directly works in mixing and production area is one of the most important factors to control.

Besides microbiological analysis, syrup, tablet and capsule as well as ointments samples were subjected for antimicrobial activity testing against some laboratory freeze dried pathogenic bacterial isolates. Some samples showed no activity at all and some showed good antibacterial activity up to $24 \mathrm{~mm}$. Vibrio spp. and Salmonella spp. showed best results with the drugs and Enterobacter spp. showed the least results. As Vibrio spp. and Salmonella spp. both have some pathogenic species, they showed good results by showing larger zone of inhibition. Antibacterial activity against other bacteria was also found out to be average activity of around $15 \mathrm{~mm}$. As all of the oral drugs (syrup, tablet and capsule) possess some antibacterial activity, the products themselves are contaminated with some bacteria which fall under the same genera of classification but the species might be different so that the presence of bacteria and the inhibiting bacteria might be affected differently by these drugs. Same scenario is applicable for ointments as well. There is less risk with contamination as these products are not administered orally and the route of entry of all the bacteria might not be skin. But while applying the ointments in broken skin with exposed blood, the bacteria might get disseminated within the body with blood circulation.

\section{Conclusion}

Present study revealed a huge amount of heterotrophic microbes as well as some specific bacteria which are 


\section{Open Access Journal of Microbiology \& Biotechnology}

beyond the accepted limit according to USP (United States Pharmacopeia) and BP (British Pharmacopeia). However, stringent regulatory actions on the microbiological quality control along with the personal hygienic improvement during formulation, handling, and storage of products would be effective for the better management of overall public health situation. Besides strict regulation and law enforcement must be applied by the government to control such production of bad quality pharmaceutical products.

\section{References}

1. Ratajczak M, Kubicka MM, Kaminska D, Sawicka P, Długaszewska J (2015) Microbiological quality of nonsterile pharmaceutical products. Saudi Pharm J 23(3): 303-307.

2. Hussain A, Wahab S, Zarin I, Hussain MDS (2010) Antibacterial activity of the leaves of Coccinia indica (W and A) W of India. Adv Biol Res 4(5): 241-248.

3. Mugoyela V, Mwambete KD (2010) Microbial contamination of nonsterile pharmaceuticals in public hospital settings. Therapeutics and Clinical Risk Management. 6: 443-448.

4. Kabir MS, Hossain MD (2013) Microbiological Quality Assessment of Vitamin B Syrups and Antibiotic Susceptibility Profile of the Isolated Escherichia coli. IOSR Journal of Pharmacy and Biological Sciences (IOSRJPBS) 8(4): 61-64.

5. Muhammed A, Umoh VJ (2009) Incidence and effects of microorganisms on the quality of some pharmaceutical mixtures in zaria-nigeria. Nigerian Journal of Pharmaceutical Sciences 8(2): 126-134.

6. Adeshina GO, Ajayi S, Onaolapo JA (2009) Microbial quality of some commercially available paediatric anti-malarial and cough preparations in Ilorin Nigeria. Nigerian Journal of Pharmaceutical Sciences 8(1): 109117.

7. Parida RK (2010) Overview of Pharmaceutical Validation and Process Controls in Drug Development. Der Pharmacia Sinica 1(1): 11-19.

8. Shaqra QMA, Shawaqfah MT, Momani WA (2014) Microbiological Quality of Blister Pack Tablets in Community Pharmacies in Jordan. Tropical Journal of Pharmaceutical Research 13(2): 261-266.

9. United States Pharmacopeia (USP) (2003) Microbiological examination of nonsterile products: Tests for specified microorganisms. Pharm Forum 29(5): 1722-1733.
10. Jimenez L (2007) Microbial bio burden on oral solid dosage forms. PDAJ Pharm Sci Tech. 61: 383-399.

11. Akerele JO, Ukoh GC (2002) Aspects of microbial contamination of tablets dispensed in hospitals and community pharmacies in Benin City, Nigeria. Tropical Journal of Pharmaceutical Research 1(1): 23-28.

12. Parker MS (1984) The preservation of oral dosage forms. Int J Pharm Tech Prod Mfr 5: 20-24.

13. Espicom (2012) The pharmaceutical market: Bangladesh, opportunities and challenges.

14. Das KK, Fatema KK, Nur IT, Noor R (2013) Prevalence of Microorganisms in commonly used cosmetics samples in Dhaka metropolis. J Pharma Sci Inno 2(6): 7-9.

15. Rana J, Sultana T, Das KK, Noor R (2014) Microbiological analysis of topicals available in Bangladesh. international journal of pharmacy and pharmaceutical sciences 6(2): 330-332.

16. Noor R, Saha SR, Rahman F, Munshi SK, Uddin MA, et al. (2012) Frequency of opportunistic and other intestinal parasitic infections in patients infected with human immunodeficiency virus in Bangladesh. Tzu Chi Medical Journal 24: 191-195.

17. Ahmed $T$, Islam $T$, Akhter $M$, Islam $K$ (2019) Microbiological Quality Analysis Along With The Drug Resistance Pattern Of The Identified Bacteria Of Different Types Of Locally Produced Sauces Available In Some Popular Fast Food Shops In Dhaka Metropolis. Journal of Environmental Science, Toxicology and Food Technology 13(5): 42-50.

18. Lucky NA, Nur IT, Ahmed T (2016) Microbiological quality assessment for drug resistant pathogenic microorganisms from the fresh vended fruit juices. Stamford Journal of Microbiology 6(1): 7-10.

19. Roy CR, Ahmed T, Uddin MA (2017) Microbiological analysis of poultry feeds along with the demonstration of the antibiotic susceptibility of the isolates and the antibacterial activity of the feeds. Bangladesh Journal of Microbiology 34(2): 103-107.

20. Acharjee M, Jahan F, Rahman F, Noor R (2013) Bacterial proliferation in municipal water supplied in Mirpur locality of Dhaka city, Bangladesh. Clean- Soil, Air, Water 42(4): 434-441.

21. Afroz H, Ahmed T, Uddin MA (2015) Microbiological analysis and antibacterial activity of pear samples. Stamford Journal of Microbiology 5(1): 1-4. 


\section{Open Access Journal of Microbiology \& Biotechnology}

22. Malek M, Akhter J, Ahmed T, Uddin MA (2015) Isolation and quantification of microorganisms from some common milk products within Dhaka city, Bangladesh. Stamford Journal of Microbiology 5 (1): 13-17.

23. Piangka N, Ahmed T, Acharjee M (2016) Microbiological analysis for drug resistant pathogenic microorganisms with determination of the antibacterial properties found in Fragaria $\mathrm{x}$ ananassa (strawberry) samples. Stamford Journal of Microbiology 6(1): 16-19.

24. Cappuccino JG, Sherman N (1996) Microbiology-A Laboratory Manual. The Benjamin/Cummings Publishing Co., Inc., Menlo Park, California.

25. Rahman F, Noor R (2012) Prevalence of pathogenic bacteria in common salad vegetables of Dhaka Metropolis. Bangladesh Journal of Botany41(2):159-162.

26. Ahmed T, Urmi NJ, Munna MS, Das KK, Acharjee M, et al.
(2014) Assessment of microbiological proliferation and in vitro demonstration of the antimicrobial activity of the commonly available salad vegetables within Dhaka Metropolis, Bangladesh. American Journal of Agriculture and Forestry 2(3): 55-60.

27. Jagessar RC, Mars A, Gones G (2008) Selective antimicrobial properties of leaf extract against various micro-organisms using disc diffusion and agar well diffusion method. J Nat Sci 6(2): 24-38.

28. Hossain J (2009) Importance of the bioburden test in pharmaceutical quality control. Pharmaceutical Microbiology Forum 15(1): 2-14.

29. Manu-Tawiah W, Brescia BA, Montgomery ER (2001) Setting threshold limits for the significance of objectionable microorganisms in oral pharmaceutical products. PDA J PharmSci Technol 55(3): 171-175. 